\title{
A NOVEL SELF-COLLISION DETECTION ALGORITHM FOR DUAL- ARM UPPER LIMB REHABILITATION ROBOT BASED ON KEY POINT-KEY LINE SEGMENT MODEL
}

\author{
Yingchao Huang ${ }^{1}$, Shuai Guo ${ }^{1}$, Leigang Zhang ${ }^{1}$ \\ ${ }^{1}$ School of Mechatronic Engineering and Automation, Shanghai University, China \\ E-mails: guoshuai@t.shu.edu.cn,1916197482@qq.com, lgzhang@shu.edu.cn
}

\begin{abstract}
Aiming at the self-collision of stroke patients in bilateral rehabilitation training in dualarm upper limb rehabilitation robot system, a minimum distance detection algorithm is proposed based on a key point-key line segment model in this paper. Firstly, the kinematics model of the system is carried out; secondly, the key point-key line segment model is established; thirdly, the minimum distance between two robots is calculated according to the real-time spatial position of the model in order to detect the self-collision; finally, the test is designed to verify the correctness of the algorithm. The result shows that robots become difficult to move when they are close to each other. The algorithm proposed in this paper can fully implement the detection of self-collision through experiments in different situations.
\end{abstract}

Keywords: Key Point-key Line Segment Model, Self-Collision Detection, Forward Kinematics.

\section{Introduction}

Stroke refers to persistent neurological deficits caused by acute cerebrovascular diseases [1].

Most patients have symptoms of upper limb hemiplegia and require specific rehabilitation training. The traditional one-on-one training with the help of doctors is costly and inefficient.

Therefore, robot-assisted system becomes a trend of rehabilitation training [2]. Common upper limb rehabilitation robots include MIME [3], BIManuTrackE [4], X0-UL7 [5], etc. Clinical medicine shows that bilateral rehabilitation training based on robot-assisted system has better effect on the treatment of hemiplegia caused by stroke than traditional single arm rehabilitation training [6].However, when the patient interacts with the robots in the bilateral rehabilitation training, there is a danger of self-collision of the robots, which poses a threat to the patient's personal safety [7]. Therefore, it is necessary to detect the self-collision in daily training. In response to the problem of self-collision, (Alexander D \& Thomas W \& Alin A \& Gerd H) proposed a new reactive self-collision avoidance algorithm which based on torque-based framework for highly complex robotic systems with a large number of DOF.

The experiment on the multi-DOF humanoid Justin clearly validated the performance and the real-time applicability of the algorithm [8].
(Fumi S. \& Kazuhiro K. \& Yasuhisa H) proposed two priority functions so as to consider task constraints and environmental constraints during the selfcollision avoidance motion for human-robot cooperating systems which are controlled based on the force/moment applied from a human.

The proposed motion control algorithm was implemented in MR Helper [9]. Other algorithms include force/torque frequency analysis [10] and image processing [11]. However, the method above relies on the accuracy established by the geometric model of the robot, the accuracy of the external sensor and the performance of the camera.

Aiming at the shortcomings of the detection methods above, a self-collision detection algorithm based on the key point-key line segment model is proposed in this paper.

The model is obtained by simplifying the system. According to its spatial position relationship, the minimum distance between two robots is calculated.

The structure of this paper is as follow: section 2 introduces the establishment of the system and its kinematics model; section 3 includes model simplification and minimum distance algorithm; the correctness of the algorithm is verified in the dualarm upper limb rehabilitation robot system in section 4; section 5 is the summary of this paper. 


\section{Kinematics Model of the System}

In response to the prevalence of hemiplegia in stroke patients, we have developed a dual-arm upper limb rehabilitation robot system. The healthy limb guides the impaired limb with the assistance of robot to finish bilateral training, and visual feedback was given to improve the rehabilitation efficiency. The system is shown in Figure 1, which mainly includes two 7-DOF robots and a motion capture system.

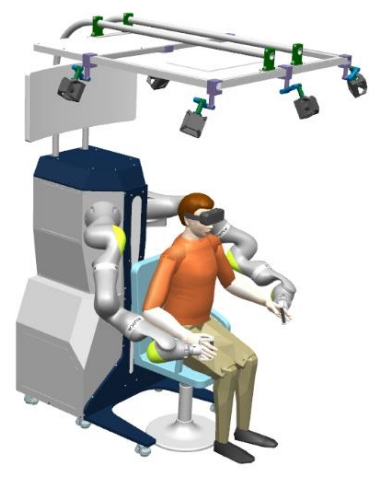

Figure 1: Dual-arm upper limb rehabilitation robot system

The workflow is as follow: firstly, the motion capture system is calibrated; secondly, the patient is required to sit in a designated position and a specified number of mark points are tied on both sides of the arm; thirdly, the upper computer control system sends a command to the robot to set the impedance control mode, and the patient interacts with the end effectors of the robot to finish bilateral training; subsequently, the motion capture system establishes the rigid model for upper limb of patient, and the motion of hand is mapped to the motion of the model in the Unity system; finally, the patient uses the actions of grabbing and throwing to complete the corresponding task.

In order to describe the position of two robots in the same coordinate system, the kinematics model of the system is required [12].

As shown below, $O_{\text {base }}$ is common coordinate system, $O_{L}$ is the basic coordinate system of left robot while $O_{R}$ is the basic coordinate system of right robot. The actual $\mathrm{Z}$ direction is perpendicular to the ground. $L=334.1 \mathrm{~mm}, \theta=45^{\circ}$.

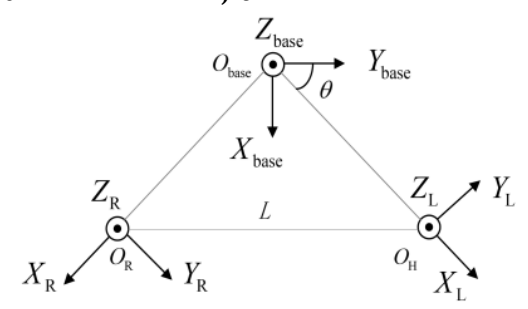

Figure 2: Schematic diagram of the robot coordinate systems
Transformation matrix from $O_{b a s e}$ to $O_{L}$ is:

$$
{ }_{L}^{\text {base }} T=\operatorname{Rot}(Z, \theta) \times \operatorname{Tran}(\mathrm{L} / 2,0,0)
$$

Similarly, matrix from $O_{\text {base }}$ to $O_{R}$ is:

$$
{ }_{R}^{\text {base }} T=\operatorname{Rot}(Z,-\theta) \times \operatorname{Tran}(\mathrm{L} / 2,0,0)
$$

The default basic coordinate system of the robot is $O_{0 L}$ rather than $O_{L}$, transformation matrix from $O_{L}$ to $O_{0 L}$ is ${ }_{0 L}^{L} T$ while transformation matrix from $O_{R}$ to $O_{0 R}$ is ${ }_{0 R}^{R} T$.

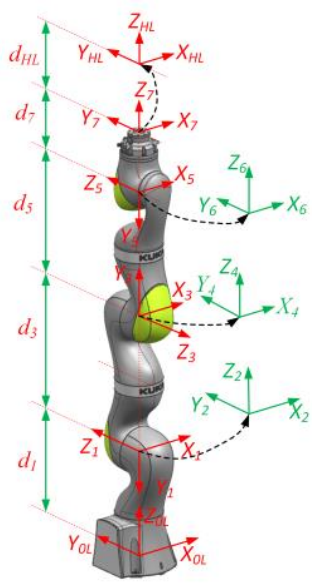

Figure 3: Robot link coordinate systems distribution diagram

Table 1. D-H Parameter table

\begin{tabular}{|c|c|c|c|c|c|}
\hline Link & $\theta_{i}$ & $\alpha_{i}$ & $a_{i}$ & $d_{i}$ & Range \\
\hline 1 & $\theta_{1}$ & -90 & 0 & 340 & $-170 \sim 170$ \\
\hline 2 & $\theta_{2}$ & 90 & 0 & 0 & $-120 \sim 120$ \\
\hline 3 & $\theta_{3}$ & 90 & 0 & 400 & $-170 \sim 170$ \\
\hline 4 & $\theta_{4}$ & -90 & 0 & 0 & $-120 \sim 120$ \\
\hline 5 & $\theta_{5}$ & -90 & 0 & 400 & $-170 \sim 170$ \\
\hline 6 & $\theta_{6}$ & 90 & 0 & 0 & $-120 \sim 120$ \\
\hline 7 & $\theta_{7}$ & 0 & 0 & 0 & $-175 \sim 175$ \\
\hline
\end{tabular}

The robot link coordinate systems distribution chart and D-H parameter table are shown above [13], $O_{H L}$ is the coordinate system of end effectors. Taking left robot as an example, transformation matrix from $O_{0 L}$ to $O_{H L}$ is ${ }_{H L}{ }_{H L}^{L}$.

The transformation from $O_{b a s e}$ to $O_{H L}$ is:

$$
{ }_{H L}^{\text {base }} T={ }_{L}^{\text {base }} T \times{ }_{0 L}^{L} T \times{ }_{H L}{ }_{H L}^{L} T
$$

Similarly, transformation from $O_{b a s e}$ to $O_{H R}$ is:

$$
{ }_{H R}^{\text {base }} T={ }_{R}^{\text {base }} T \times{ }_{0 R}^{R} T \times{ }_{H R}^{0 R} T
$$




\section{Minimum \\ Distance \\ Detection Algorithm}

During the bilateral training, the position of the robot on both sides changes in real time, and the position of each joint changes continuously, which leading to the change of the spatial position of each link [14]. Therefore, the self-collision problem is transformed into the relative spatial positional relationship between the links of two robots.

Each link of the robot can be simplified to a cylinder with a certain height, so it is simplified as a central axis, the radius of which is subsequently compensated [15]. The system is simplified into the following model:

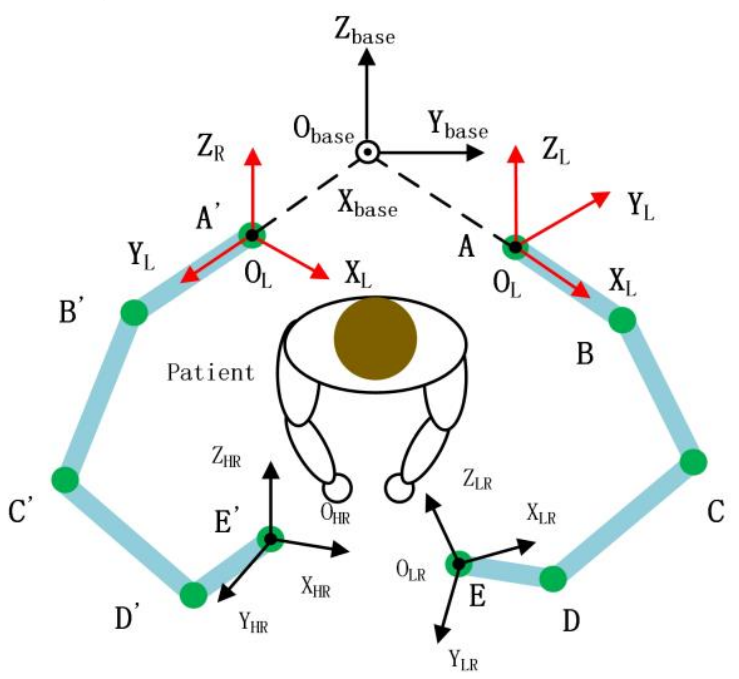

Figure 4: Initial key point-key line segment model

A, B, C, D, D, E is the key points of left robot, the rotation of joint $1,3,5$ and 7 in the model does not affect the properties of the collinearity of the adjacent links. Link 1 and link 2 are simplified into line segment $A B$, link 3 and link 4 are simplified into line segment $B C$, link 5 and link 6 are simplified into line segment $C D$, link 7 and end effector are simplified into line segment $D E$. Similarly, the right robot is simplified into key points $A^{\prime}, B^{\prime}, C^{\prime}, D^{\prime}, E^{\prime}$, and key line segments $A^{\prime} B^{\prime}, B^{\prime} C^{\prime}, C^{\prime} D^{\prime}, D^{\prime} E^{\prime}$.

The robot is mounted on the system and its central position of base $A$ or $A^{\prime}$ is stationary. Therefore, the spatial position of line segment $A B$, $A^{\prime} B^{\prime}$ is a constant, and there is no collision between them. Due to the spatial interference of the patient and the range of each joint angle, $B C, C D, D E$ is difficult to interfere with $A^{\prime} B^{\prime}$ while $B^{\prime} C^{\prime}, C^{\prime} D^{\prime}, D^{\prime} E^{\prime}$ is difficult to interfere with $A B$, so the system is further simplified to the following model:

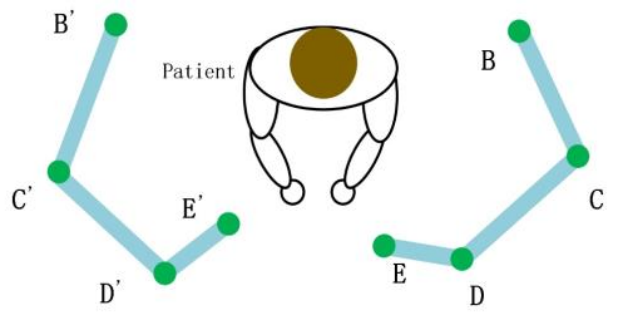

Figure 5: Final key point-key line segment model

Under this model, there are three types of selfcollision situations.

a. End effector collides with link:

B'

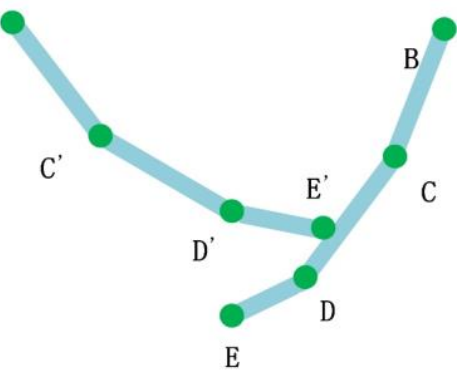

Figure 6: Collision situation 1

b. Link collides with link:

$\mathrm{B}^{\prime}$

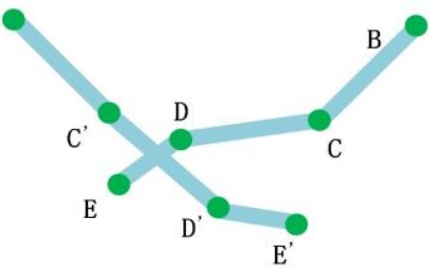

Figure 7: Collision situation 2

c. End effector collides with end effector:

$\mathrm{B}^{\prime}$

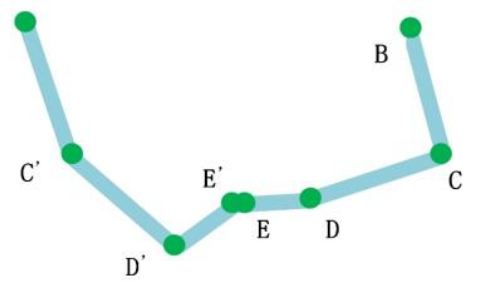

Figure 8: Collision situation 3

Therefore, the self-collision problem is transformed into the minimum distance between left and right robot in the model. Distances of $B C$ and $B^{\prime} C^{\prime}, B C$ and $C^{\prime} D^{\prime}, B C$ and $D^{\prime} E^{\prime}, C D$ and $B^{\prime} C^{\prime}, C D$ and $C^{\prime} D^{\prime}, C D$ and $D^{\prime} E^{\prime}, D E$ and $B^{\prime} C^{\prime}, D E$ and $C^{\prime} D^{\prime}, D E$ and $D^{\prime} E^{\prime}$ are required to calculate.

Now the problem is turned into spatial distance between $i t h(1 \leq i \leq 3)$ line segment of left robot and $j t h(1 \leq j \leq 3)$ line segment of right robot. 
Taking $B C$ and $C^{\prime} D^{\prime}$ as an example, as shown below:

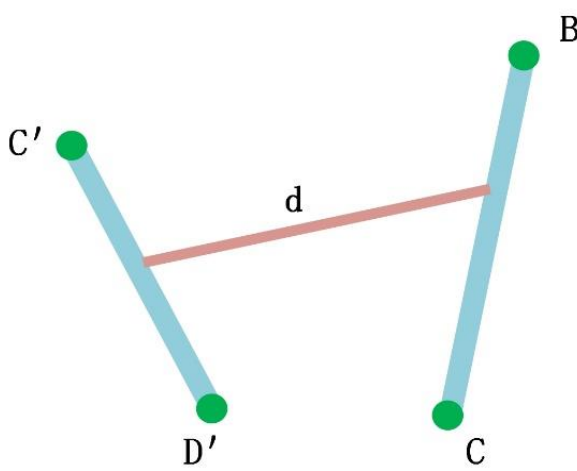

Figure 9: Schematic diagram of the distance between the spatial line segments

To calculate spatial distance $d$, it is necessary to determine the spatial position of each key line segment while key line segment is determined by two adjacent key points. So the spatial position of the key point $B, C, C^{\prime}, D^{\prime}$ is required to determine.

Taking left robot as an example, $B$ corresponds to joint 2, $C$ corresponds to joint $4, D$ corresponds to joint 6, $E$ corresponds to end effectors. The position of the joint $n$ relative to $O_{\text {base }}$ is as follow:

$$
\begin{aligned}
& { }_{n}^{\text {base }} T={ }_{L}^{\text {base }} T \times{ }_{0 L}^{L} T \times{ }_{n}{ }_{n} T \\
& ={ }_{L}^{\text {base }} T \times{ }_{0 L}^{L} T \times{ }_{1}^{0 L} T \times{ }_{2}^{1} T \times \ldots \times{ }_{n}^{n-1} T \\
& =\left[\begin{array}{cccc}
{ }_{n}^{\text {base }} \boldsymbol{R} & & { }^{\text {base }} \boldsymbol{P} \\
0 & 0 & 0 & 1
\end{array}\right]=\left[\begin{array}{cccc}
r_{11} & r_{12} & r_{13} & x_{n} \\
r_{21} & r_{22} & r_{23} & y_{n} \\
r_{31} & r_{32} & r_{33} & z_{n} \\
0 & 0 & 0 & 1
\end{array}\right]
\end{aligned}
$$

$\left(x_{n}, y_{n}, z_{n}\right)$ is the position of joint $n$.

Similarly, the position of end effectors $\left(x_{H L}, y_{H L}, z_{H L}\right)$ is

$$
{ }_{H L}^{\text {base }} T={ }_{L}^{\text {base }} T \times{ }_{0 L}^{L} T \times{ }_{7} T \times{ }_{H L}^{7} T
$$

Key line segment $i$ is determined by $\left(x_{i}, y_{i}, z_{i}\right)$ and $\left(x_{i+1}, y_{i+1}, z_{i+1}\right)$, the coordinate is shown as follow:

$$
\left(x_{i+1}-x_{i}, y_{i+1}-y_{i}, z_{i+1}-z_{i}\right)
$$

After determining the spatial position of the key points and the key line segments, the distances between all key line segments at each moment can be calculated.

$B C$ and $C^{\prime} D^{\prime}$ are equally divided into $m$ and $n$ parts respectively to obtain the following model.

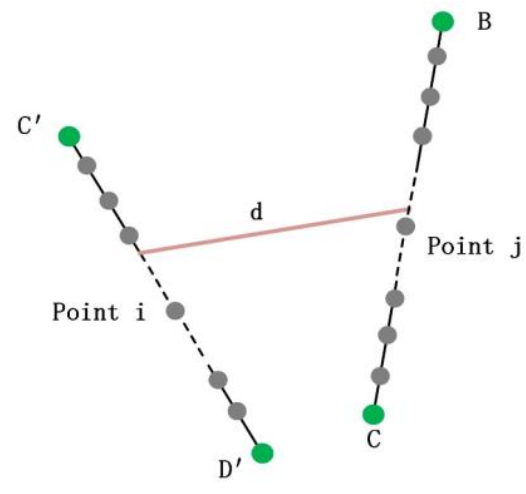

Figure 10: Schematic diagram of spatial line segments distance calculation

$B C$ is determined by $B\left(x_{B}, y_{B}, z_{B}\right)$ and $C\left(x_{C}, y_{C}, z_{C}\right)$ while $C^{\prime} D^{\prime}$ is determined by $C^{\prime}\left(x_{C^{\prime}}, y_{C^{\prime}}, z_{C^{\prime}}\right)$ and $D^{\prime}\left(x_{D^{\prime}}, y_{D^{\prime}}, z_{D^{\prime}}\right)$.Position of the ith point $P i\left(P i_{x}, P i_{y}, P i_{z}\right)$ is:

$\operatorname{Pi}\left(x_{B}+\frac{\left(x_{C}-x_{B}\right)}{m} \times(i-1), y_{B}+\frac{\left(y_{C}-y_{B}\right)}{m}\right.$
$\left.\times(i-1), z_{B}+\frac{\left(z_{C}-z_{B}\right)}{m} \times(i-1)\right)$

where $(1 \leq i \leq m+1)$.

Position of the $j t h$ point $P j\left(P j_{x}, P j_{y}, P j_{z}\right)$ on $C^{\prime} D^{\prime}$ is:

$$
\begin{aligned}
& P j\left(x_{C^{\prime}}+\frac{\left(x_{D^{\prime}}-x_{C^{\prime}}\right)}{n} \times(j-1), y_{C^{\prime}}+\frac{\left(y_{D^{\prime}}-y_{C^{\prime}}\right)}{n}\right. \\
& \left.\times(j-1), z_{C^{\prime}}+\frac{\left(z_{D^{\prime}}-z_{C^{\prime}}\right)}{n} \times(j-1)\right)
\end{aligned}
$$

where $(1 \leq j \leq n+1)$.

distance of $P i$ and $P j$ is:

$\mathrm{d}_{i j}=\sqrt{\left(P i_{x}-P j_{x}\right)^{2}+\left(P i_{y}-P j_{y}\right)^{2}+\left(P i_{z}-P j_{z}\right)^{2}}$

$(m+1) \times(n+1)$ distances are required to calculate to obtain the minimum distance between two-line segments. The distances between 9 pairs of line segments above need to be calculated and the minimum distance of the model is received.

The key line segments in the model ignore the actual radius of robot, so $\mathrm{d}_{i j}$ needs to be compensated.

By measuring the radius of the robot links, the maximum one is chosen as the compensation radius $r$. 


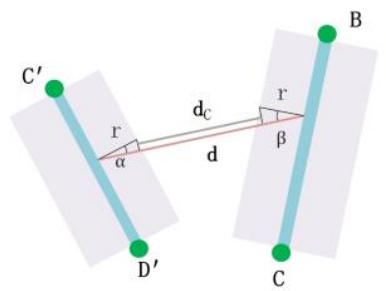

Figure 11: Schematic diagram of radius Compensation

As shown above, after considering the radius of the robot, there is an inequality:

$$
d-\mathrm{r} \cos \alpha-r \cos \beta \geq d-r-r
$$

Therefore, it will be safer to take $d_{c}=d-2 r$ as the minimum detection distance after compensation. Self-collision is detected by comparing $d_{f}$ and $d_{c}$.

\section{Test}

In order to verify the effectiveness and safety of the self-collision algorithm based on the key point-key line segment model that may exist in the system, a healthy person test is designed to ask the tester to perform task-oriented training in a collaborative training mode.
Data such as resultant force of the end effectors, the position of each joint and end effectors are acquired in real time.

Data sharing is required between two robots. The higher frequency of accepting data ensures the realtime nature of the test. $d_{c}$ is calculated according to joint angles $\left[\theta_{1}, \theta_{2}, \theta_{3}, \theta_{4}, \theta_{5}, \theta_{6}, \theta_{7}\right]$ of both sides.

In order to demonstrate the effect of collision detection algorithm, impedance control parameters are set for the robots. When $d_{c}$ is greater than $d_{f}$, Cartesian stiffness is set as $\mathrm{K}_{\mathrm{x}}=\mathrm{K}_{\mathrm{y}}=\mathrm{K}_{\mathrm{z}}=100$, when $d_{c}$ is less than $d_{f}$, Cartesian stiffness is set as $\mathrm{K}_{\mathrm{x}}=\mathrm{K}_{\mathrm{y}}=\mathrm{K}_{\mathrm{z}}=3000$.

According to Hooke's law $F=K \Delta X$, it requires a large amount of force from the tester to move the robots close to each other. When the self-collision is detected, the distance between two robots is reduced more and more difficult, the end effectors will be subjected to a large force.

As shown, the tester moves the robot to get close to each other in motion $I(I 1-I 4) 、 I I(I I 1-I I 4)$ and III (III1-III4) with different initial poses.
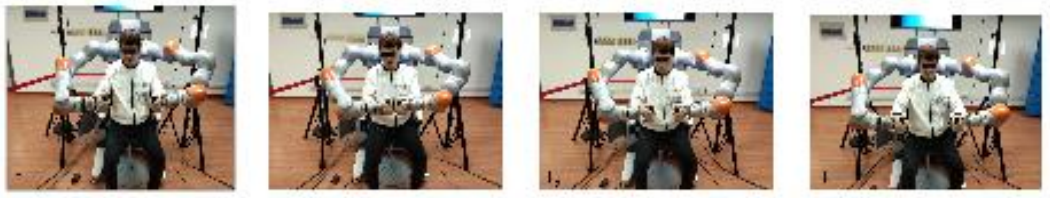

\section{(a) motion $I$}
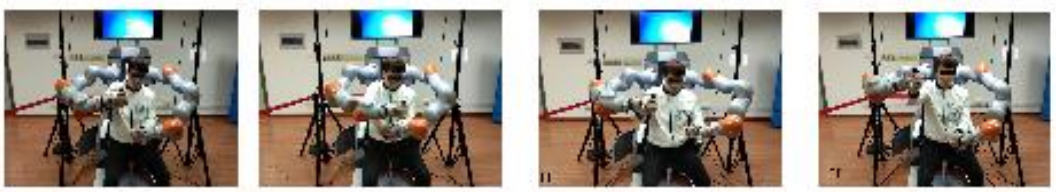

(b) motion II
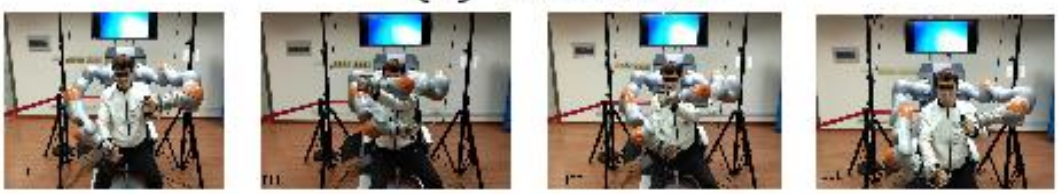

\section{(c) motion III}

Figure 12: The tester manipulates the robot in different initial poses

Obtaining the data of the robot from initial position to end position in the motion above, and the data format is: time of motion, spatial position of $B$, $C, D, E, B^{\prime}, C^{\prime}, D^{\prime}, E^{\prime}$ based on $O_{\text {base }}$, the resultant force of the end effector, the minimum distance between two robots.
In Matlab, schematic diagram of spatial position of the model、minimum distance graph and resultant force figure of end effector in three motions is drawn as follow. 

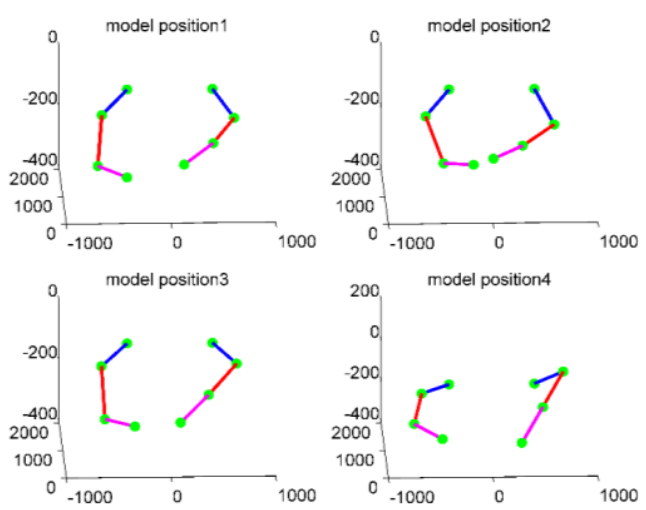

Figure 13: Schematic diagram of spatial position of model in motion $I$

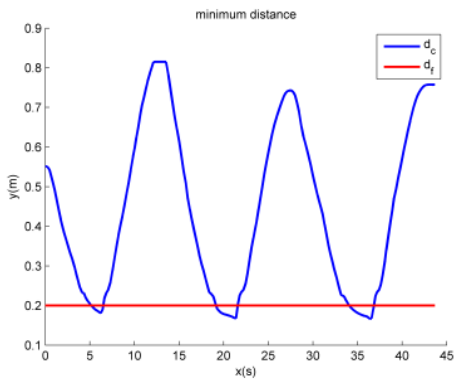

Figure 14: Minimum distance graph in motion I
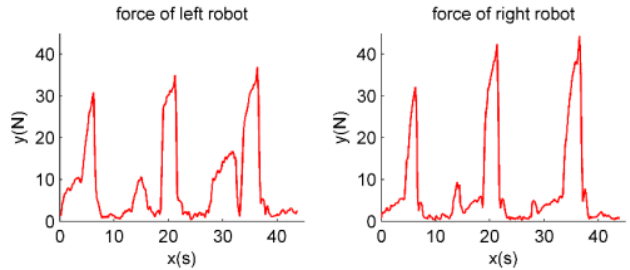

Figure 15: Resultant force figure of end effector in motion I

For motion $I, d_{c}$ changes from $0.55 m$ to $0.18 m$ during $t=0 \sim 6 s$, the resultant force on the end effector of left and right robot is constantly increasing. When $t=6 \mathrm{~s}$, the force of left robot reaches the maximum value $F_{\text {left }}=3214 \mathrm{~N}$ while the right robot reaches the maximum value $F_{\text {right }}=30.85 \mathrm{~N}$. The first self-collision was successfully detected. $d_{c}$ keeps growing while the resultant force of both left and right robots is continuously reduced during $t=6 \sim 12 s, d_{c}$ is in a safe range. The curve also satisfies the rules above during $t=12 \sim 22 \mathrm{~s}$ and $t$ $=22 \sim 37 \mathrm{~s}$.

The same method is used to analyse the results of motion II and motion III . The analysis diagram is shown below.
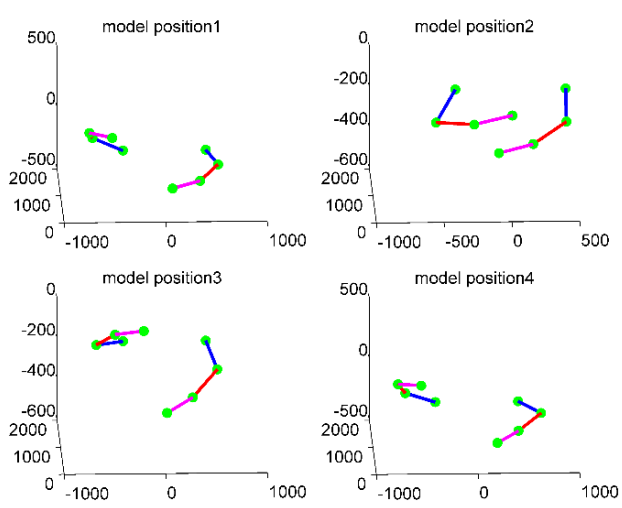

Figure 16: Schematic diagram of spatial position of model in motion II

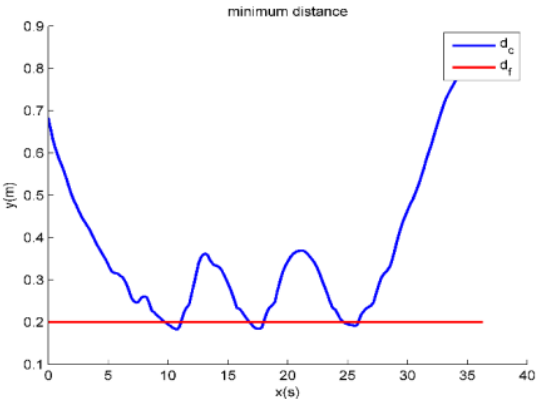

Figure 17: Minimum distance graph in motion II
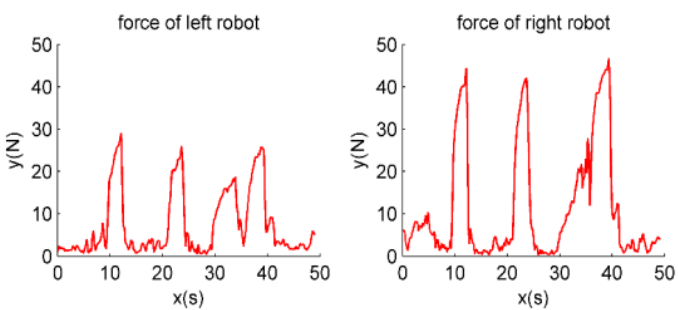

Figure 18: Resultant force figure of end effector in motion II
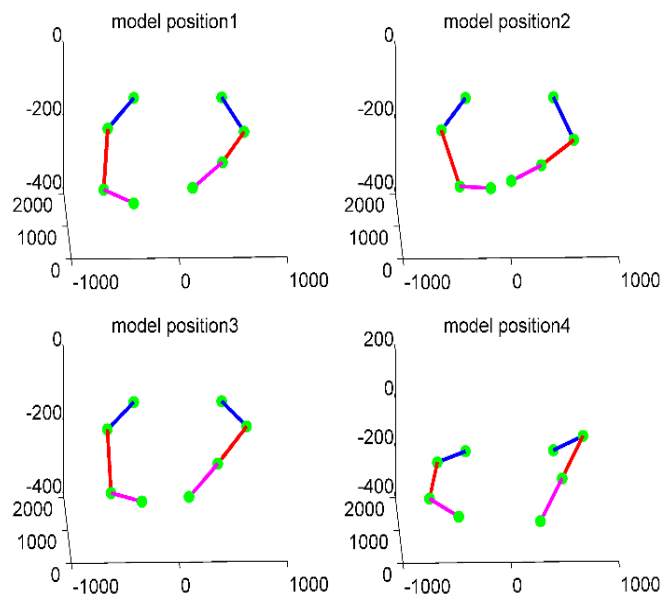

Figure 19: Schematic diagram of spatial position of model in motion III 


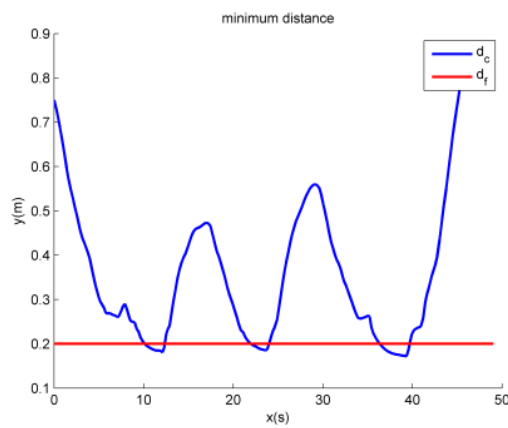

Figure 20: Minimum distance graph in motion III
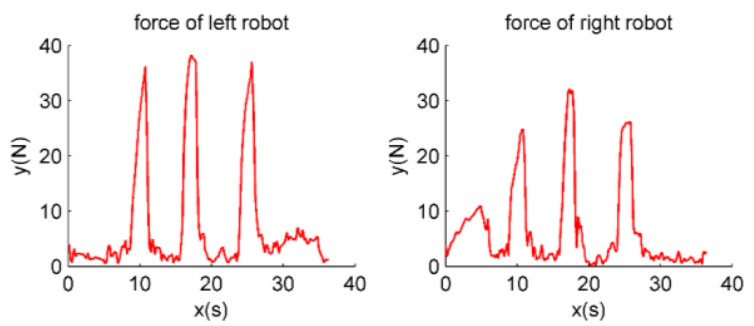

Figure 21: Resultant force figure of end effector in motion III

According to the Matlab analysis figures above, the minimum distance detection algorithm based on the key point-key line segment model is tested in view of three kinds of self-collision situation mentioned in the paper. The self-collision is about to happen when $d_{f}<d_{c}$, the resultant force of end effectors increases and it is more difficult for two robots to move closer to each other. This phenomenon verifies the effectiveness and security of the algorithm.

\section{Conclusions}

Aiming at the self-collision that may occur in the dual-arm upper limb rehabilitation robot, a minimum distance detection algorithm based on the key point-hey line segment model is proposed in this paper. By building forward kinematics model and model simplification of the system, the spatial coordinates of the joints corresponding to the key points are obtained to determine the spatial position of all key line segments. The minimum distance of the model can be calculated according to the position of the key line segment. Self-collision is detected by comparing $d_{f}$ and $d_{c}$. The test is designed to obtain data from healthy person for analysis and processing, self-collision is expressed in the form of the resultant force of the end effector, which verifies the correctness of the proposed algorithm.

\section{References}

[1] Lloydjones D, Adams R J, Brown T M, et al. Heart disease and stroke statistics--2010 update: a report from the American Heart Association. [J]. Circulation, 2010, 127(1):143-52.

[2] Kwakkel G. Intensity of leg and arm training after primary middle-cerebral-artery stroke: a randomized trial [J]. Lancet, 1999, 354.

[3] Lum P S, Burgar C G, Shor P C. Use of the MIME robotic system to retrain multijoint reaching in post-stroke hemiparesis: why some movement patterns work better than others [C]//International Conference of the IEEE Engineering in Medicine \& Biology Society. IEEE, 2003.

[4] Robertson J V G, Jarrasse N, Roby-Brami A. Rehabilitation robots: A compliment to virtual reality [J]. Schedae, 2010, 6(1): 77-94.

[5] Yu W, Rosen J. A novel linear PID controller for an upper limb exoskeleton [C]//IEEE Conference on Decision and Control. Piscataway, USA: IEEE, 2010: 3548-3553.

[6] Chan B L, Witt, Richard, Charrow, Alexandra P, et al. Mirror Therapy for Phantom Limb Pain [J]. New England Journal of Medicine, 2007, 357(21):2206-2207.

[7] Miao Q, Mcdaid A, Zhang M, et al. A three-stage trajectory generation method for robot-assisted bilateral upper limb training with subjectspecific adaptation [J]. Robotics \& Autonomous Systems, 2018.

[8] Dietrich A, Wimbock T, Albu-Schaffer A, et al. Integration of Reactive, Torque-Based SelfCollision Avoidance Into a Task Hierarchy [J]. IEEE Transactions on Robotics, 2012, 28(6):1278-1293.

[9] Dietrich A, Wimböck, Thomas, Täubig, Holger, et al. Extensions to Reactive Self-Collision Avoidance for Torque and Position Controlled Humanoids [C]// IEEE International Conference on Robotics \& Automation. IEEE, 2011.

[10] Kouris A, Dimeas F, Aspragathos N. Contact distinction in human-robot cooperation with admittance control [C]//IEEE International Conference on Systems. 2017.

[11] Petric T, Zlajpah L. [IEEE 2011 IEEE International Conference on Robotics and Biomimetics (ROBIO) - Karon Beach, Thailand (2011.12.7-2011.12.11)] $2011 \quad$ IEEE International Conference on Robotics and Biomimetics - Smooth transition between tasks on a kinematic control level: Application to self collision avoidance for two Kuka LWR robots [J]. 2011:162-167. 